Pacific Journal of Mathematics

ERGODICITY IN AFFINE SKEW-PRODUCT MORAL 


\title{
ERGODICITY IN AFFINE SKEW-PRODUCT TORAL EXTENSIONS
}

\author{
HaRvey B. Keynes ANd Mahesh G. NeruRKar
}

\begin{abstract}
We examine the dynamics of those skew-product extensions in which the fibre is a torus supporting a group of automorphisms which intertwine with the given $\mathbf{Z}^{n}$ action. The main results concern lifting ergodicity generically from the base when the original action is perturbed into new action by a continuous affine cocyle. Extensions are indicated for other properties, such as weak-mixing and Bernoulli, and smooth variants are stated.
\end{abstract}

1. Introduction. A (topological) dynamical system is a pair $(Y, T)$, where $T$ is a locally compact separable group, acting continuously on the right of the compact metric space $Y$, with action $(y, t) \rightarrow y \cdot t$. If in addition, we have a $T$ invariant Borel probability $\mu$ on $Y$, we denote this system by $(Y, T, \mu)$.

Let $(X, T)$ be another dynamical system such that $\pi:(X, T) \rightarrow(Y, T)$ is an affine extension with the fibre group $G$ supporting an automorphism group $\left\{\sigma_{t} \mid t \in T\right\}$. Let $\tilde{\mu}$ be the Haar lift of $\mu$ to $X$. (See [4] for more details on these definitions and the definitions given below.) A continuous affine cocycle $\alpha$ for $\pi$ is a continuous map $\alpha: Y \times T \rightarrow G$ satisfying the cocycle condition,

$$
\alpha\left(y, t_{1} t_{2}\right)=\alpha\left(y, t_{1}\right)\left[\sigma_{t_{1}^{-1}} \alpha\left(y \cdot t_{1}, t_{2}\right)\right], \quad \forall y, t_{1}, t_{2} .
$$

Let $Z(Y, T, G)$ be the set of all such cocycles. Given $f \in C(Y, G)$, the space of continuous maps from $Y$ to $G$, and setting $1^{f}(y, t)=$ $f(y)^{-1}\left[\sigma_{t^{-1}} f(y \cdot t)\right]$ we get a cocycle $1^{f} \in Z(Y, T, G)$, called an affine coboundary. The set of all affine coboundaries will be denoted by $B(Y, T, G)$. Given $\phi \in Z(Y, T, G)$ and $1^{f} \in B(Y, T, G)$, if

$$
\phi \cdot 1^{f}(y, t)=f(y)^{-1} \phi(y, t) \sigma_{t^{-1}}[f(y \cdot t)],
$$

then $\phi \cdot 1^{f} \in Z(Y, T, G)$. Two cocycles $\phi_{1}, \phi_{2}$ are cohomologous if $\phi_{2}=$ $\phi_{1} \cdot 1^{f}$ for some $f$.

As a subset of $C(Y \times T, G)$, we can restrict the compact-open topology to $Z(Y, T, G)$. This topology can be seen to be generated by a complete separable metric. When $T=\mathbf{Z}$, one can identify $Z(Y, T, G)$ with 
$C(Y, G)$ and the metric can be taken to be the usual supremum metric. We use the letter $d$ to denote metric on any space.

Given $\alpha \in Z(Y, T, G)$, we define the skew-product $T$ action on $X$ by setting $x \circ t=\left(\alpha(y, t)^{-1} x\right) \cdot t$ if $\pi x=y$. This new dynamical system will be denoted by $\left(X, T_{\alpha}\right)$ and is called the skew-product extensions of $Y$ by $\alpha$. Note that $(Y, T)$ is still a dynamical factor and $\tilde{\mu}$ is invariant under skew-product action but $\pi$ need not be an affine extension (e.g., if $G$ is nonabelian).

Our main results are concerned with lifting ergodicity generically to the skew-product extension.

The precise statements are given below.

TheOREM (1.1). Let $\pi:(X, T, \tilde{\mu}) \rightarrow(Y, T, \mu)$ be an affine extension with fibre $G$. Further assume that

(i) $T=\mathbf{Z}^{r}$ and $\mu$ is nonatomic

(ii) $G=\mathbf{T}^{c}$, the $c$ torus for some $c \in \mathbf{N}$ and $\sigma_{1}, \sigma_{2} \cdots, \sigma_{r}$ be automorphisms of $G$ generating an equicontinuous $\mathbf{Z}^{r}$-action.

(iii) $(Y, T, \mu)$ is ergodic.

Then the set $\left\{\alpha \mid \alpha \in \overline{B(Y, T, G)}\right.$ and $\left(X, T_{\alpha}, \tilde{\mu}\right)$ is ergodic $\}$ is residual in $\overline{B(Y, T, G)}$.

THeOREM (1.2). Let $\pi:(X, T, \tilde{\mu}) \rightarrow(Y, T, \mu)$ satisfy the conditions of Theorem (1.1) except that the $\mathbf{Z}^{r}$-action on $G$ need not be equicontinuous. Then the set $\left\{\alpha \mid \alpha \in Z(Y, T, G)\right.$ and $\left(X, T_{\alpha}, \tilde{\mu}\right)$ is ergodic $\}$ is residual in $Z(Y, T, G)$.

Notice that if we give up the periodicity of the fibre automorphisms, we need to enlarge the class to all cocycles.

We will prove these theorems for integer actions (i.e. $T=\mathbf{Z}$ ), since the proof in the general case is essentially the same. Thus we denote by $T$ the homeomorphisms both on $X$ and $Y$ generating the $\mathbf{Z}$ action and by $\sigma$ the group automorphism generating the action on $G$. The proof of Theorem (1.2) will be built in two steps. First we prove Theorem (1.1). We then complete the proof of (1.2) by modifying the constructions used to prove (1.1) and using a "Furstenberg type" tower for the automorphism $\sigma$. The details are given in $\S 2$.

\section{Proofs.}

A. Proof of (1.1). The condition of equicontinuity of $\sigma$ is equivalent to assuming that $\sigma^{p}=1$, for some $p>0$, since $G$ is a torus. First we describe briefly a useful "diamond diagram" for equicontinuous affine 
extensions, (see [5] for details.) Let $K$ be the enveloping semi-group of $(G, \sigma)$.

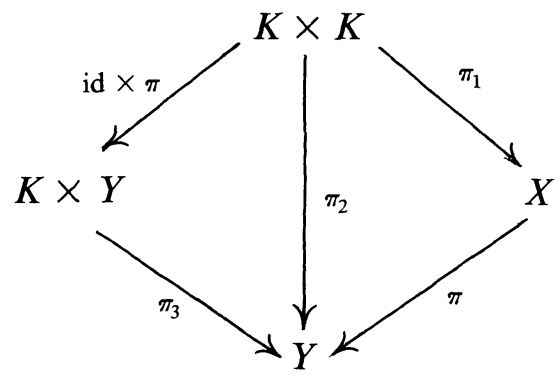

All spaces in the diagram are dynamical systems with $\mathbf{Z}$ actions and all maps are factor maps. The action on $K \times X$ is generated by homeomorphism $T(k, x)=(k \sigma, T x)$. There is a left $G$ action on $K \times X$, defined by $g(k, x)=(k, k(g) x)$, which commutes with this $\mathbf{Z}$ action. We will think of $L^{2}(X, \tilde{\mu})$ as a closed subspace of $L^{2}(K \times X, \nu \times \tilde{\mu})$, where $\nu$ is the normalized Haar measure on $K$.

We define operators on $L^{2}(K \times X, \nu \times \tilde{\mu})$ as follows.

(1) Given $\alpha \in Z(Y, T, G) \equiv C(Y, G)$, set

$$
U_{T}^{\alpha} f(k, x)=f\left(k \sigma, T\left(\alpha(y)^{-1} x\right)\right), \quad \text { where } \pi x=y .
$$

(2) Given $\psi \in C(Y, G)$, set

$$
H_{\psi} f(k, x)=f\left(k, \psi(y)^{-1} x\right) .
$$

(3) Let $P$ and $C$ be the projections onto the space of $T$ invariant functions and constant functions respectively.

(4) Set

$$
W_{n}^{\alpha}=\frac{1}{n} \sum_{i=0}^{n-1}\left(U_{T}^{\alpha}\right)^{i} .
$$

Given $f \in L^{2}(X, \tilde{\mu}), \varepsilon>0$ and $m \in \mathbf{N}$, we define

$$
\begin{aligned}
W(f, \varepsilon, m)=\{\phi \mid \phi \in \overline{B(Y, T, G)} & \text { such that } \exists M \in \mathbf{N}, M>m \\
& \text { such that } \left.\left|\left\langle W_{M}^{\phi} f, f\right\rangle-\langle C f, f\rangle\right|<\varepsilon\right\},
\end{aligned}
$$

where $\langle\cdot, \cdot\rangle$ denotes the inner product in $L^{2}(K \times X, \nu \times \tilde{\mu})$.

Using standard arguments (see [7] for related results in group extensions) it is easy to show that the proof of the theorem reduces to showing that for $f \in L^{2}(X, \tilde{\mu})$ each $W(f, \varepsilon, m)$ is open and dense in $\overline{B(Y, T, G)}$. Openess is routine and the denseness will be established in a series of lemmas below.

Lemma (2.1). Let $f \in L^{2}(X, \tilde{\mu}), \varepsilon>0$ and $m \in \mathbf{N}$. Let $\phi \in Z(Y, T, G)$ and $\psi \in C(Y, G)$. Then $\phi \cdot 1^{\psi} \in W(f, \varepsilon, m)$ iff $\phi \in W\left(H_{\psi} f, \varepsilon, m\right)$. 
Proof. The proof follows from observing that, (i) $W_{n}^{\alpha} H_{\psi}=H_{\psi} W_{n}^{\alpha \cdot 1^{\psi}}$ and (ii) $\mathrm{CH}_{\psi}=\mathrm{H}_{\psi} \mathrm{C}=\mathrm{C}$

Lemma (2.2). Assume that for any $f \in L^{2}(X, \tilde{\mu}), \varepsilon>0, m \in \mathbf{N}$, given $\delta>0$, there exist $\psi_{\delta} \equiv \psi \in C(Y, G)$ such that

(i) $D\left(1^{\psi}, 1\right)<\delta$ and

(ii) $1^{\psi} \in W(f, \varepsilon, m)$.

Then for any $f, \varepsilon>0$ and $m \in \mathbf{N}, W(f, \varepsilon, m)$ is dense in $\overline{B(Y, T, G)}$. Here, $D$ is the metric defined by $D\left(\phi_{1}, \phi_{2}\right)=\sup _{y \in Y} d\left(\phi_{1}(y), \phi_{2}(y)\right)$.

Proof. The condition in this lemma implies that $1 \in \overline{W(f, \varepsilon, m)}$ $\forall f, \varepsilon, m$. Now the previous lemma implies $1^{\psi} \in \overline{W(f, \varepsilon, m)}, \forall f, \varepsilon, m$. This immediately implies $\overline{W(f, \varepsilon, m)}=\overline{B(Y, T, G)}$.

From this point onwards, we will assume in addition that the bundle $\pi:(X, T) \rightarrow(Y, T)$ is "dynamically trivial". This means that $X=G \times Y$ and $T(g, y)=(\sigma(g), T y)$. We define additional operators $U_{T}^{0}$ and $V$ on $L^{2}(K \times X) \equiv L^{2}(G \times K \times Y)$ by setting,

$$
\begin{aligned}
& U_{T}^{0} f(g, k, y)=f(g, k \sigma, T y) \text { and } \\
& V f(g, k, y)=f\left(k^{-1}(g), k, y\right) .
\end{aligned}
$$

Note that when $\alpha=1, U_{T}^{1} f(g, k, y)=f(\sigma(g), k \sigma, T y)$, so $U_{T}^{0}$ disregards the action of $\sigma$ on $G$. Set

$$
W_{n}^{0}=\frac{1}{n} \sum_{i=0}^{n-1}\left(U_{T}^{0}\right)^{i} .
$$

Then the following lemma can be easily verified.

$$
\text { LeMma (2.3) (i) } W_{n}^{1} \circ V=V \circ W_{n}^{0}, \forall n \text { and (ii) } V C=C V=C \text {. }
$$

Next, we view the flow $T(k, x)=(k \sigma, T x)$ on $K \times X$ as a $K$ skewproduct extension of $(Y, T, \mu)$ by the cocycle $(y, n) \rightarrow \sigma^{n}(n \in \mathbf{Z})$. Then applying a result of $\mathbf{R}$. Zimmer (see [9]) to this cocylce we get a Borel map $\zeta: Y \rightarrow K$ and a closed subgroup $K_{0}$ of $K$ such that (i) $\tilde{\sigma}(y, n)=$ $\zeta(y)^{-1} \sigma^{n} \zeta\left(T^{n} y\right) \in K_{0}, \forall(y, n) \in Y \times \mathbf{Z}$ and (ii) $\left(K_{0} \times_{\tilde{\sigma}} Y, \nu_{K_{0}} \times \mu\right)$ is ergodic, where $\nu_{K_{0}}$ is the normalized Haar measure on $K_{0}$. setting,

The restriction to $K_{0}$ yields operators $B$ and $P_{K_{0}}$ on $L^{2}(K \times X)$ by

(i) $B f(g, k, y)=f(g, k(y), y)$ and

(ii) $P_{K_{0}}(g, k, y)=\int_{Y} \int_{K_{0}} f\left(g, k k_{0}, y\right) d \nu_{K_{0}}\left(k_{0}\right) d \mu(y)$. 
Note again that $B C=C B=B$

LEMMA (2.4) The operators $W_{n}^{0}$ converge weakly to $B^{-1} P_{K_{0}} B$ as $n \rightarrow \infty$.

Proof. This is essentially a restatement of the above result of Zimmer. For an explicit proof, see [7].

Lemma (2.5). Given $f \in L^{2}(X, \tilde{\mu}), \varepsilon>0, \delta>0$, suppose that there exists $\psi \in C(Y, G)$ such that,

(a) $D\left(1^{\psi}, 1\right)<\delta$ and

(b) $\left\|P_{K_{0}} B V^{-1} H_{\psi} f-C f\right\|<\varepsilon$.

Then $W(f, \varepsilon, m)$ is dense in $\overline{B(Y, T, G)}$, for any $f, \varepsilon$ and $m$.

Proof. From Lemma (2.2), given $m \in \mathbf{N}$, we need to find $\psi \in C(Y, G)$ such that (i) $D\left(1^{\psi}, 1\right)<\delta$ and (ii) $1^{\psi} \in W(f, \varepsilon, m)$. Now for any $\psi$,

$$
\begin{aligned}
\left\langle W_{n}^{1^{\psi}} f, f\right\rangle & =\left\langle H_{\psi} W_{n}^{1^{\psi}} f, H_{\psi} f\right\rangle=\left\langle W_{n}^{1} H_{\psi} f, H_{\psi} f\right\rangle \\
& =\left\langle V W_{n}^{0} V^{-1} H_{\psi} f, H_{\psi} f\right\rangle=\left\langle W_{n}^{0} V^{-1} H_{\psi} f, V^{-1} H_{\psi} f\right\rangle .
\end{aligned}
$$

Therefore,

$$
\begin{aligned}
\left|\left\langle W_{n}^{1^{\psi}} f, f\right\rangle-\langle C f, f\rangle\right| & \\
\leq & \left|\left\langle W_{n}^{0} V^{-1} H_{\psi} f, V^{-1} H_{\psi} f\right\rangle-\left\langle B^{-1} P_{K_{0}} B V^{-1} H_{\psi} f, V^{-1} H_{\psi} f\right\rangle\right| \\
& +\left|\left\langle B^{-1} P_{K_{0}} B V^{-1} H_{\psi} f, V^{-1} H_{\psi} f\right\rangle-\langle C f, f\rangle\right| .
\end{aligned}
$$

The 2 nd term is equal to

$$
\begin{aligned}
& \left|\left\langle B^{-1} P_{K_{0}} B V^{-1} H_{\psi} f, V^{-1} H_{\psi} f\right\rangle-\left\langle V^{-1} H_{\psi} C f, V^{-1} H_{\psi} f\right\rangle\right| \\
& \quad=\left|\left\langle B^{-1} P_{K_{0}} B V^{-1} H_{\psi} f, V^{-1} H_{\psi} f\right\rangle-\left\langle C f, V^{-1} H_{\psi} f\right\rangle\right| \\
& \quad \leq\left\|B^{-1} P_{K_{0}} B V^{-1} H_{\psi} f-C f\right\|\left\|V^{-1} H_{\psi} f\right\| \\
& \left.\quad \leq\left\|B^{-1}\right\|\left\|V^{-1} H_{\psi}\right\|\|f\|\left\|P_{K_{0}} B V^{-1} H_{\psi} f-C f\right\| \quad \text { (since } B^{-1} C=C\right) .
\end{aligned}
$$

Thus given $\varepsilon>0$, condition (b) allows us to pick $\psi \in C(Y, G)$ such that the 2 nd term is less than $\varepsilon / 2$. Then (2.4) allows us to select $M$ large enough so that the first term in (*) is also less than $\varepsilon / 2$. This yields (ii) and completes the proof.

The proof of (1.1) now reduces to the following result.

Lemma (2.6). Given any $f \in L^{2}(X, \tilde{\mu}), \varepsilon>0$ and $\delta>0$, there exists $\psi \in C(Y, G)$ such that (i) $D\left(1^{\psi}, 1\right)<\delta$ and (ii) $\left\|P_{K_{0}} B V^{-1} H_{\psi} f-C f\right\|<\varepsilon$. 
Proof. Without loss of generality, we assume $f$ to be continuous. Let $M=\sup _{y \in Y}|f(y)|$. Let $\eta^{\prime}>0$ be a small number to be specified later. Let $h: \mathbf{R}^{c} \rightarrow \mathbf{T}^{c}$ be $h\left(t_{1}, t_{2}, \ldots, t_{c}\right)=\left(e^{2 \pi i t_{1}}, \ldots, e^{2 \pi t t_{c}}\right)$ and $A$ a $c \times c$ nonsingular matrix with integer entries corresponding to $\sigma$ (this implies that $\left.\sigma(h x)=h(A x), \forall x \in \mathbf{R}^{c}\right)$. Pick $\delta_{1}>0$ such that

$$
\text { if } x, y \in \mathbf{R}^{c} \text { and }\|x-y\|<\delta_{1} \text {, then } d(h(x), h(y))<\delta .
$$

Then pick $N \in \mathbf{N}$ such that,

(i) $\quad N=k p$ for some $k$, and

(ii) $2 p \sup _{-p \leq i \leq p}\left\|A^{i}\right\|<N \delta_{1}$, and $\frac{N}{N^{2}-1}<\frac{\eta^{\prime}}{M}$.

Now using Rokhlin's lemma we get a Borel set $V \subseteq Y$ such that, $V, T V, \ldots, T^{N^{2}-1} V$ are pairwise disjoint

$$
\text { and } \mu\left(Y-\bigcup_{i=0}^{N^{2}-1} T^{l} V\right)<\frac{\eta^{\prime}}{M} \text {. }
$$

Next, let $\alpha>0$ be such that, if $y_{1}, y_{2} \in Y$ and $d\left(y_{1}, y_{2}\right)<\alpha$ then

$$
\left|f\left(g, T^{l} y_{1}\right)-f\left(g, T^{i} y_{2}\right)\right|<\eta^{\prime}, \quad \forall g \in G \text { and } 0 \leq i \leq N^{2}-1 .
$$

Further partition $V$ into disjoint Borel sets $B_{i}(1 \leq i \leq R+1)$ where,

$$
\begin{aligned}
& \operatorname{diam}\left(B_{i}\right)<\alpha, \mu\left(B_{i}\right)>0, \quad \forall 1 \leq i \leq R, \quad \mu\left(B_{R+1}\right)=0 \\
& \text { and the map } \zeta \text { is constant on each } B_{i} .
\end{aligned}
$$

Since $\zeta$ takes only finitely many values this is possible. Let $I=[0,1]$ and $I^{c p}=I^{c} \times I^{c} \times \cdots \times I^{c}(p$ times $)$. Let $l$ denote Lebesgue measure on $[0,1]$. Since each $B_{l}$ is standard Borel and $\mu$ is nonatomic, we let $\psi^{l}$ : $B_{\imath} \rightarrow\left(I^{c p}, \mathscr{B}_{I^{c p}}\right)$ be a Borel isomorphism such that,

$$
\left(\psi^{i}\right)_{*}\left(\left.\mu\right|_{B_{i}}\right)=l^{c p}, \quad \forall 1 \leq i \leq R .
$$

Set $\psi^{R+1} \equiv 0$ on $B_{R+1}$.

We can write $\psi^{i}(y)=\left(\psi_{j}^{i}(y)\right)_{0 \leq j \leq p-1}$ where $\psi_{j}^{i}: B_{l} \rightarrow I^{c}$ is the projection of $\psi^{i}$ on the $j$ th component. Next, set

$$
\begin{aligned}
& \tilde{\psi}\left(T^{j} y\right)=p \psi_{s}^{i}(y), \quad \forall y \in B_{i} \text { where } j \equiv s \bmod p \\
& \qquad\left(0 \leq j \leq N^{2}-1,0 \leq s \leq p-1\right) .
\end{aligned}
$$

Then setting $\tilde{\psi} \equiv 0$ outside $\bigcup_{l=0}^{N^{2}-1} T^{\imath} V$, we get a Borel map $\tilde{\psi}:\left(Y, \mathscr{B}_{Y}\right) \rightarrow$ $[0, p]^{c}$. 
By using Lusin's approximation, we can select a continuous map $\theta$ : $Y \rightarrow[0, p]^{c}$ such that,

$$
\mu\{y \mid \theta(y) \neq \tilde{\psi}(y)\}<\frac{\eta^{\prime}}{N^{2} M}
$$

We then define,

$$
\tilde{\theta}(y)=\frac{1}{N} \sum_{i=0}^{N-1}\left(A^{i}\right)^{-1} \theta\left(T^{i} y\right) .
$$

Clearly, $\tilde{\boldsymbol{\theta}}: Y: \rightarrow \mathbf{R}^{c}$ is a bounded continuous map. Finally, set $\psi=h \circ \theta$. We will now show that $\psi$ is the required map.

To see that condition (i) is satisfied, it is enough to check by our choice of $\delta_{1}$ in (1) that $\sup _{y \in Y}\|\tilde{\theta}(T y)-A \tilde{\theta}(y)\|<\delta_{1}$. But this last condition holds since,

$$
\begin{aligned}
\| \tilde{\theta}(T y)- & A \tilde{\theta}(y)\left\|=\frac{1}{N}\right\| \sum_{i=0}^{N-1}\left(A^{i}\right)^{-1} \theta\left(T^{i+1} y\right)-\sum_{i=0}^{N-1} A^{-i+1} \theta\left(T^{i} y\right) \| \\
=\frac{1}{N} \| \theta(T y)+A^{-1} \theta\left(T^{2} y\right)+\cdots+A^{-(N-1)} \theta\left(T^{N} y\right)-A \theta(y) & -\theta(T y)-A^{-1} \theta\left(T^{2} y\right) \cdots \| \\
\leq \frac{1}{N} \cdot 2 \sup _{-p \leq i \leq p}\left\|A^{i}\right\| p<\delta_{1}, & \text { using }(2) .
\end{aligned}
$$

We next consider condition (ii). We first note that

$$
\begin{gathered}
P_{K_{0}} B V^{-1} H_{\psi} f(g, k, y)=\int_{K_{0} \times Y}\left(B V^{-1} H_{\psi} f\right)\left(g, k k^{\prime}, y\right) d \nu_{K_{0}}\left(k^{\prime}\right) \times d \mu(y) \\
=\int_{K_{0} \times Y} f\left(\left[k k^{\prime} \zeta(y)\right] g \psi(y), k k^{\prime} \zeta(y), y\right) d \nu_{K_{0}}\left(k^{\prime}\right) \times d \mu(y) .
\end{gathered}
$$

Now since $f \in L^{2}(X, \tilde{\mu}), f$ depends only on the $G$ and $Y$ components. Hence,

$$
P_{K_{0}} B V^{-1} H_{\psi} f(g, k, y)=\int_{K_{0}} \int_{Y} f\left(\left[k k^{\prime} \zeta(y)\right] g \psi(y), y\right) d \mu(y) d \nu_{K_{0}}\left(k^{\prime}\right) .
$$

We now pick $y_{i} \in B_{l}, 1 \leq i \leq p$, and fix them throughout the remainder of the proof. By (4) and (5) it follows that, $\forall k, k^{\prime}, g$ we have

$$
\begin{aligned}
\left|\int_{B_{i}} f\left(\left[k k^{\prime} \zeta(y)\right] g \psi(y), y\right) d \mu\right|_{B_{i}}(y) \\
\quad-\left.\int_{B_{i}} f\left(\left[k k^{\prime} \zeta\left(y_{i}\right)\right] g \psi(y), y_{i}\right) d \mu\right|_{B_{i}}(y) \mid<\eta^{\prime} .
\end{aligned}
$$


Let $B_{i}^{0}=\left\{y \mid y \in B_{i}, \tilde{\theta}(y) \neq \tilde{\psi}(y)\right\}$. Then using (8) and (9) it follows that,

$$
\mu\left(B_{i}^{0}\right)<\frac{N \eta^{\prime}}{N^{2} M}<\frac{\eta^{\prime}}{M} .
$$

Also, if $y \in B_{i}-B_{i}^{0}$, then

$$
\begin{aligned}
\psi(y) & =h\left(\frac{1}{N} \sum_{j=0}^{N-1} A^{-\jmath} \tilde{\psi}\left(T^{j} y\right)\right)=h\left(\frac{1}{p} \sum_{i=0}^{p-1} A^{-j} p \psi_{j}^{i}(y)\right) \cdots \quad(\text { using }(7)) \\
& =\prod_{j=0}^{p-1} \sigma^{-j} h\left(\psi_{j}^{i}(y)\right) .
\end{aligned}
$$

We now examine $G^{p}=G \times G \times \cdots \times G$ ( $p$ times), and the following maps,

$$
\begin{gathered}
\bar{h}=h \times h \times \cdots \times h: I^{c p} \rightarrow G^{p} \quad(p \text { times }), \\
\bar{\sigma}=\sigma^{-(p-1)} \times \sigma^{-(p-2)} \times \cdots \times \sigma^{0}: G^{p} \rightarrow G^{p}, \\
\chi\left(g_{1}, g_{2} \cdots g_{p}\right)=g_{1} g_{2} \cdots g_{p} ; \chi: G^{p} \rightarrow G .
\end{gathered}
$$

Then letting “ " denote "within $\eta^{\prime}$ of”, we obtain

$$
\begin{aligned}
\left.\int_{B_{l}} f\left(\left[k k^{\prime} \zeta\left(y_{\imath}\right)\right] g\left(\prod_{j=0}^{p-1} \sigma^{-j} h \psi_{j}^{i}(y)\right), y_{j}\right) d \mu\right|_{B_{\imath}}(y) \\
\left.\quad \sim \int_{B_{i}} f\left(\left[k k^{\prime} \zeta\left(y_{i}\right)\right] g \chi \circ \bar{\sigma} \circ \bar{h} \psi^{i}(y), y_{i}\right) d \mu\right|_{B_{\imath}}(y) \\
\quad \sim \int_{I^{c p}} f\left(\left[k k^{\prime} \zeta\left(y_{i}\right)\right] g \chi \circ \bar{\sigma} \circ \bar{h}(t), y_{\imath}\right) d l^{c p}(t) \\
\sim \int_{G^{p}} f\left(\left[k k^{\prime} \zeta\left(y_{i}\right)\right] g \chi(\bar{g}), y_{\imath}\right) d \eta^{p}(\bar{g}), \\
\quad \eta^{p}=\eta \times \eta \times \cdots \times \eta(p \text { times }) \\
\sim \int_{G} f\left(g^{\prime}, y_{i}\right) d \eta\left(g^{\prime}\right) .
\end{aligned}
$$

Combining these estimates yields,

$$
\begin{aligned}
& \mid \int_{B_{i}} f\left(\left[k k^{\prime} \zeta(y)\right] g \psi\right.(y), y) d \mu(y)-\mu\left(B_{i}\right) \int_{G} f\left(g^{\prime}, y_{l}\right) d \eta\left(g^{\prime}\right) \mid \\
&<5 \eta^{\prime} \mu\left(B_{i}\right) .
\end{aligned}
$$


By using (7), it is clear that this type of estimate will hold for integrals taken over $T^{s} B_{i}$, where $0 \leq s \leq N^{2}-N-1$ and $1 \leq i \leq R$. Moreover, from (3) and our choice of $N$ in (ii) of (2) remaining set has measure $\leq 2 \eta^{\prime} / M$. This implies

$$
\begin{gathered}
\left|P_{K_{0}} B V^{-1} H_{\psi} f(g, k, y)-\sum_{i=1}^{R} \sum_{j=0}^{N^{2}-1} \mu\left(B_{i}\right) \int_{G} f\left(g, T^{j} y_{i}\right) d \eta(g)\right| \\
<7 \eta^{\prime}, \quad \forall(g, k, y) .
\end{gathered}
$$

From this it follows that $\left\|P_{K_{0}} B V^{-1} H_{\psi} f-C f\right\|<\varepsilon$ if $\eta^{\prime}$ is chosen sufficiently small. This completes the proof under dynamical triviality.

B. Completion of (1.1) and proof of (1.2). We now give an argument that (i) shows the assumption of dynamical triviality of the bundle $\pi: X \rightarrow Y$ can be removed and (ii) extend the proof of (1.1) with no assumption on $\sigma$, to the class $Z(Y, T, G)$ of all cocycles. In the case the $\pi$ is not trivial, by using a Borel section we can assume that the $T$ action on $X$ is generated by $T(g, y)=\left(\sigma(g \phi(y)), T_{y}\right)$ for some Borel map $\phi$ : $Y \rightarrow G$. To prove (1.2), we will repeat the same arguments replacing $B(Y, T, G)$ by $Z(Y, T, G)$. In either case we finally reduce the proof to showing that for any Borel $\phi: Y \rightarrow G$, given $\delta, \varepsilon>0, m \in \mathbf{N}$ and $f \in L^{2}(X, \tilde{\mu})$ we can find $\psi \in C(Y, G)$ such that

(i) $D\left(1^{\psi}, 1\right)<\delta$ and

(ii) $\left|\left\langle W_{M}^{\phi \cdot 1^{\psi}} f, f\right\rangle-\langle C f, f\rangle\right|<\varepsilon$ for some $M>m$.

This is analog of Lemma (2.2). To prove the result needed we define the operator $V_{\alpha}(\alpha \in Z(Y, T, G))$ by setting

$$
V_{\alpha} f(g, k, y)=\left(k^{-1}(\phi(y) g), k, y\right) \text {. }
$$

Then one can readily verify that $W_{n}^{1} \circ V=V_{\phi} \circ W_{n}^{0}$. Using this instead of the previous relation $\left(W_{n}^{1} \circ V=V \circ W_{n}^{0}\right)$ as appropriate and the fact that $V_{\phi} C=C$ in Lemma (2.5), one can directly verify that we come up with the same conditions (namely $D\left(1^{\psi}, 1\right)<\delta$ and $\left\|P_{K_{0}} B V^{-1} H_{\psi} f-C f\right\|<\varepsilon$ ). This proves Theorem (1.2) still under the assumption that $\sigma$ is periodic.

We now consider the case of a general $\sigma$. Let $H \subseteq G$ be a closed $\sigma$-invariant subgroup such that $\sigma$ factored onto the quotient group $H \backslash G$ is equicontinuous. Consider the affine extension $\pi_{1}: X_{1}=H \backslash X \rightarrow Y$ with fibre $H \backslash G$, obtained by factoring by $H$. Then one can prove that the set of $\alpha \in Z(Y, T, G)$ for which the skew product flow induced on $X_{1}$ by $\alpha$ is ergodic, is a residual subset of $Z(Y, T, G)$. This proceeds in the following steps: we can repeat Lemmas (2.1) through (2.5) for this class of cocycles, and the construction of $\psi$ in Lemma (2.6) can be easily modified so that $\psi$ in addition factors through $H$. We replace our $h$ by the map 
$\beta \circ \tilde{h}$, where $\beta: G \rightarrow H \backslash G$ is the factor map and $\tilde{h}: I^{p c} \rightarrow G$ is the exponential map. Note that $\beta \circ \tilde{h}$ takes the Lebesgue measure onto the normalized Haar measure on $H \backslash G$ and this is precisely what is really needed in Lemma (2.6). The general proof of (1.2) is obtained by selecting our subgroup $H$ properly. The following result tells us precisely what our $H$ should be.

Proposition (2.7) ([5]). Let $\pi:(X, T, \tilde{\mu}) \rightarrow(Y, T, \mu)$ be an affine extension with fibre group $G$.

(1) Let $(Y, T, \mu)$ be ergodic. Then $(X, T, \tilde{\mu})$ is ergodic (weakly-mixing) iff the affine bi-transformation group $\left(G_{F} \backslash G, G_{F} \backslash X, T, \bar{\mu}\right)$ is ergodic (weakly mixing). Here $\bar{\mu}=\left(\pi_{1}\right)_{*} \tilde{\mu}, \pi_{1}: X \rightarrow G_{F} \backslash X$ is the quotient map and $G_{F} \subseteq G$ is a closed $\sigma$-invariant subgroup such that $\left(G_{F} \backslash G, \sigma\right)$ is the maximal equicontinuous factor of $(G, \sigma)$.

(2) Furthermore if $(G, \sigma)$ is distal, and $(Y, T, \mu)$ is uniquely ergodic then $(X, T, \tilde{\mu})$ is uniquely ergodic iff it is ergodic.

So choosing $H=G_{F}$ in the previous argument finishes the proof of Theorem (1.2).

REMARK. The question arises whether Theorem (1.2) can be obtained without the enlargement to the class of all cocycles. If the automorphism $\sigma$ is distal but not equicontinuous, our proof does not enable us to lift ergodicity generically in the class of closure of coboundaries. The main obstacle is the lack of an analog of the "diamond diagram" (see §2.A for a more detailed definition). This diagram is critical even in the equicontinuous $\left(\sigma^{p}=I\right)$ case, and enables us to view $\sigma$ itself as a cocycle into $K$ - the enveloping semigroup (see Lemma 2.3). In turn, this observation helps us to lift ergodicity without any additional assumptions like ergodicity of $\left(Y, T^{p}, \mu\right)$. However, we do not know if Theorem (1.2) is true for distal actions in the closure of the coboundaries.

In the case that $\sigma$ is ergodic, it seems likely that the closure of $\sigma$-coboundaries should be the set of all cocycles. In the measure theoretic situation D. Lind has proven that for ergodic $\sigma$, every $\sigma$-cocycle is a measurably a $\sigma$ couboundary. It seems possible that some topological variants of his techniques might yield this result. Note that when $G / G_{F}$ is trivial, for example, when $\sigma$ is hyperbolic, then every cocycle yields an ergodic action.

3. Extensions. Several extensions of (1.2) follow readily. One can replace ergodicity in Theorem (1.2) by weakly mixing. This is proved by 
using the product bundle $X \times X \stackrel{\pi \times \pi}{\rightarrow} Y \times Y$. Using the same techniques, we can prove a generic theorem for lifting ergodicity in the class $\{\alpha \times \alpha \mid$ $\alpha \in Z(Y, T, G)\} \subseteq Z(Y \times Y, T, G \times G)$. In addition, a result of $\mathrm{D}$. Rudolph (see [8]) shows that for affine extensions with Bernoulli base, the skew product flow is Bernoulli iff it is weakly mixing. These observations can be summarized in the following theorem.

TheOREM (3.1) Let $\pi:(X, T, \tilde{\mu}) \rightarrow(Y, T, \mu)$ be an affine extension as in Theorem (1.2).

(i) If $(Y, T, \mu)$ is weakly-mixing (Bernoulli), then $\{\alpha \mid \alpha \in Z(Y, T, G)$ such that $\left(X, T_{\alpha}, \tilde{\mu}\right)$ is weakly mixing (Bernoulli) $\}$ is residual in $Z(Y, T, G)$.

(ii) If $(G, \sigma)$ is distal then ergodicity in (1.2) can be replaced by unique ergodicity.

Part (ii) is an immediate consequence of (2) of Proposition (2.7).

Some additional properties can be added to the properties lifted as in Theorem (3.1), (i). Results of Parry [7a] show that $K$-automorphisms are lifted. Unpublished results of Thovenot and Rudolph yield that mixing and $k$-fold mixing, respectively, are also lifted.

Finally, we examine some smooth versions of these results. Suppose $Y$ is a compact connected $C^{\infty}$ manifold and $T: Y \rightarrow Y$ is a $C^{\infty}$ diffeomorphism, preserving a smooth measure $\mu$. In this general setting it is unknown whether any smooth version of Theorem (1.2) holds. The main difficulty in applying these techniques in the smooth case arises in Lemma (2.6). In general, it is not possible to construct a smooth map $\psi: Y \rightarrow G$ satisfying (i) $D_{1}\left(1^{\psi}, 1\right)<\delta$ and (ii) $\left\|P B V^{-1} H_{\psi} f-C f\right\|<\varepsilon$, where $D_{1}$ is the $C^{1}$ metric on $C^{1}$ maps from $Y$ to $G$. However, adopting a technique used in [6], under the special situation of an irrational rotation on a circle, one can prove a smooth analog of Lemma (2.6) by putting restrictions on irrational rotation number $\alpha$. The following is the precise statement.

THEOREM (3.2). Let $\pi: X \rightarrow Y$ be an affine extension as in Theorem (1.2), where $(Y, T)$ is an irrational rotation on the circle. Assume that there exists a sequence $\left(p_{n} / q_{n}\right)$ of irreducible fractions such that $\left|\alpha-p_{n} / q_{n}\right| \leq$ $M / q_{n}^{2+\gamma}$ where $M>0, \gamma>0$ are constants and $q_{n} \rightarrow \infty$ an $n \rightarrow \infty$. Then the set of $\phi \in C^{1}(Y, G)$ such that $\left(X, T_{\phi}, \mu\right)$ is ergodic is residual in $C^{1}(Y, G)$.

The above theorem in the context of group extensions yields a smooth version of a result of Glasner and Weiss in [3]. For more general 
diffeomorphisms $T: Y \rightarrow Y$, it seems possible that smooth versions of Theorem (1.2) may hold under the assumption that $T$ admits periodic approximations with sufficient speed.

\section{REFERENCES}

[1] M. Denker, C. Grillenberger, K. Sigmund, Ergodic Theory on Compact Spaces, Springer Verlag L. N. \#527.

[2] H. Furstenberg, Strict ergodicity and transformations of the torus, Amer. J. Math., 83 (1961), 573-601.

[3] S. Glasner and B. Weiss, On the construction of minimal skew products, Israel J. Math., 34 (1979).

[4] H.Keynes and D. Newton, Minimal $(G, \sigma)$ extensions, Pacific J. Math., 77 (1978), 145-163.

[5] _ Ergodicity in $(G, \sigma)$ Extensions, Proc. Inter. Conf. on Dyn. systems, Springer Verlag L. N. 819 p. 265.

[6] M. Nerurkar, Recurrent-Proximal linear differential systems with almost periodic coefficients (submitted).

[7] Generic theorems for continuous ergodic skew product actions of amenable groups, Pacific J. Math., 119, no. 2, (1985).

[7a] W. Parry, Ergodic properties of affine transformations and flows on nilmanifolds, Amer. J. Math., 91 (1969), 757-771.

[8] D. Rudolph, Classifying the isometric extensions of a Bernoulli shift, J. Analyse Math., 34 (1978), 36-60.

[9] R. Zimmer, Extensions of ergodic group actions, Illinois J. Math., 20 (1976), 373-409.

Received October 15, 1984 and in revised form May 16, 1985. Research partially supportd by N. S. F. Grant MCS 81-02034.

\section{UNIVERSITY OF MINNESOTA}

MINNEAPOLIS, MN 55455

AND

OHIo STATE UNIVERSITY

Columbus, OH 43210 


\section{PACIFIC JOURNAL OF MATHEMATICS EDITORS}

V. S. VARADARAJAN

(Managing Editor)

University of California

Los Angeles, CA 90024

HERBERT CLEMENS

University of Utah

Salt Lake City, UT 84112

R. FINN

Stanford University

Stanford, CA 94305
HERMANN FLASCHKA

University of Arizona

Tucson, AZ 85721

RAMESH A. GANGOLLI

University of Washington

Seattle, WA 98195

VAUGHAN F. R. JONES

University of California

Berkeley, CA 94720

ROBION KIRBY

University of California

Berkeley, CA 94720
C. C. MOORE

University of California

Berkeley, CA 94720

H. SAMELSON

Stanford University

Stanford, CA 94305

HAROLD STARK

University of California, San Diego

La Jolla, CA 92093

\section{ASSOCIATE EDITORS}

R. ARENS

E. F. BECKENBACH

B. H. NEUMANN (1906-1982)
F. WOLF

K. YOSHIDA

\section{SUPPORTING INSTITUTIONS}

UNIVERSITY OF ARIZONA

UNIVERSITY OF BRITISH COLUMBIA

CALIFORNIA INSTITUTE OF TECHNOLOGY

UNIVERSITY OF CALIFORNIA

MONTANA STATE UNIVERSITY

UNIVERSITY OF NEVADA, RENO

NEW MEXICO STATE UNIVERSITY

OREGON STATE UNIVERSITY
UNIVERSITY OF OREGON UNIVERSITY OF SOUTHERN CALIFORNIA

STANFORD UNIVERSITY

UNIVERSITY OF HAWAII

UNIVERSITY OF TOKYO

UNIVERSITY OF UTAH

WASHINGTON STATE UNIVERSITY

UNIVERSITY OF WASHINGTON 


\section{Pacific Journal of Mathematics}

\section{Vol. 123, No. $1 \quad$ March, 1986}

Maria Emilia Alonso García, A note on orderings on algebraic varieties $\ldots \ldots 1$

F. S. De Blasi and Józef Myjak, On continuous approximations for

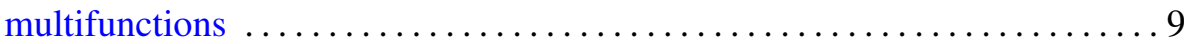

Frank Albert Farris, An intrinsic construction of Fefferman's CR metric . . . 33 Antonio Giambruno, P. Misso and Francisco César Polcino Milies, Derivations with invertible values in rings with involution $\ldots . \ldots . \ldots .47$

Dan Haran and Moshe Jarden, The absolute Galois group of a pseudo real

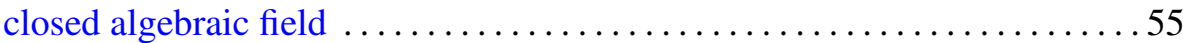

Telemachos E. Hatziafratis, Integral representation formulas on analytic

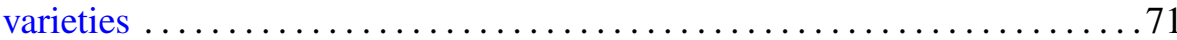

Douglas Austin Hensley, Dirichlet's theorem for the ring of polynomials

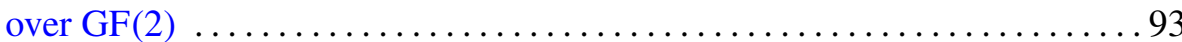

Sofia Kalpazidou, On a problem of Gauss-Kuzmin type for continued

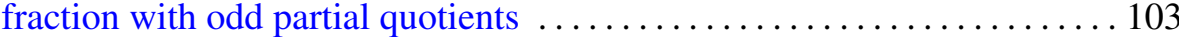

Harvey Bayard Keynes and Mahesh Nerurkar, Ergodicity in affine

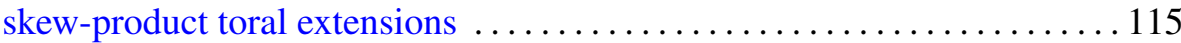

Thomas Landes, Normal structure and the sum-property $\ldots \ldots \ldots \ldots \ldots 127$

Anthony To-Ming Lau and Viktor Losert, Weak*-closed complemented invariant subspaces of $L_{\infty}(G)$ and amenable locally compact groups ...149 Andrew Lelek, Continua of constant distances in span theory . . . . . . . . 161 Dominikus Noll, Sums and products of $B_{r}$ spaces $\ldots \ldots \ldots \ldots \ldots \ldots \ldots \ldots$ Lucimar Nova, Fixed point theorems for some discontinuous operators 189

A. A. S. Perera and Donald Rayl Wilken, On extreme points and support points of the family of starlike functions of order $\alpha$

Massimo A. Picardello, Positive definite functions and $L^{p}$ convolution operators on amalgams ........................

Friedrich Roesler, Squarefree integers in nonlinear sequences ......... 223

Theodore Shifrin, The osculatory behavior of surfaces in $\mathbf{P}^{5}$ 